\title{
Three novel members of angucycline group from Streptomyces sp. N05WA963
}

\author{
Xiao Ren, Xinhua Lu, Aibing Ke, Zhihui Zheng, Jie Lin, Weili Hao, Jingtong Zhu, Yuling Fan, Yanbo Ding, \\ Qin Jiang and Hua Zhang
}

Three novel members of angucycline family named N05WA963A (1), B (2) and D (4), together with a new anthracycline named N05WA963C (3) were isolated from the culture broth of Streptomyces sp. N05WA963. The structures were elucidated on the basis of comprehensive spectral data analysis. All four compounds have shown antiproliferative effects on a panel of cancer cell lines such as SW620, K-562, MDA-MB-231, YES-4, T-98 and U251SP.

The Journal of Antibiotics (2011) 64, 339-343; doi:10.1038/ja.2011.4; published online 2 February 2011

Keywords: angucyclines; anthracycline; antiproliferative effects; Streptomyces sp. N05WA963

\section{INTRODUCTION}

In the course of our screening program for novel antitumor antibiotics, a fermentation extract of Streptomyces sp. N05WA963 was assayed by a 3-(4, 5-dimethyl-2-thiazolyl)-2, 5-diphenyl-2H-tetrazolium bromide (MTT) method testing for the growth inhibitory activity on the colon cancer cell line SW620. From the production of the strain, four antibiotics named N05WA963A (1), B (2), C (3) and $\mathrm{D}(\mathbf{4})$ were purified as the active compounds. Three of them $(\mathbf{1}, 2$, 4) are urdamycin B-type angucyclines having a C-glycosidic moiety without angularic oxygens. ${ }^{1}$ Whereas, the compound $\mathbf{3}$ with a linearly condensed ring system belongs to the anthracycline group, and has the same aglycone as an irradiation product of aquayamycin. ${ }^{2}$ This paper describes the fermentation, isolation, physical properties, structure elucidation and biological activities of these compounds.

\section{RESULTS}

\section{Taxonomy}

The strain N05WA963 was grown on yeast extract-malt extract agar (ISP2). The vegetative mycelia were brownish yellow and did not show fragmentation into coccoid forms or bacillary elements. The grey aerial mycelia grew abundantly and formed spiral spora chain. The sequence of $16 \mathrm{~S}$ ribosomal DNA confirmed the strain as a Streptomyces species showing a homology of $>99 \%$ with type strains of this genus.

\section{Physicochemical properties}

Each compound was soluble in methanol, ethanol, acetone, chloroform and ethyl acetate, but insoluble in water, $n$-hexane and petroleum ether. The other physical properties of these compounds are listed in Table 1. The general features of their UV and NMR spectra resembled each other, especially compound 1,2 and 4 , indicating structural similarities of these compounds.

Structure determination of N05WA963A (1)

N05WA963A (1) was obtained as a dark yellow powder. The UV spectrum of 1 with maxima at $\lambda_{\max } 220,285$ and $399 \mathrm{~nm}$ in $\mathrm{MeOH}$ closely resembled those reported for urdamycin $\mathrm{B},{ }^{3}$ the representative of angucycline group. The high-resolution FAB-MS (m/z $709.2871[\mathrm{M}+\mathrm{H}]^{+}$, calcd for $709.2860, \Delta 1.1 \mathrm{mmu}$ error) suggested its molecular formula was $\mathrm{C}_{38} \mathrm{H}_{44} \mathrm{O}_{13}$. The signal for a phenolic proton at 12.60 p.p.m. in downfield region of the ${ }^{1} \mathrm{H}$ NMR spectrum, as well as the signals at $\delta 188.0$ and $\delta 181.7$ in the ${ }^{13} \mathrm{C}$ NMR spectrum indicated the location of the hydroxyl group at the $\alpha$-position of anthraquinone. In addition, one AB-type system $(\delta 7.87$ and $7.68, J=7.0 \mathrm{~Hz})$ and a singlet at $\delta 7.67$ deduced substitutions at the 4a-, 5-, 8-, 9- and 12b-position of the anthraquinone skeleton. The assumption was confirmed by its HMBC correlations from OH-8 $(\delta 12.60)$ to C-8, 9, 7, 7a, H-6 (87.67) to C-4a, 5, 6a, 7, 12a, H-11 (87.68) to C-7a, 11a, 12, 9, 10 and $\mathrm{H}-10(\delta 7.87)$ to $\mathrm{C}-8,11,11 \mathrm{a}$. In another way, its HMBC correlations from $\mathrm{H}_{2}-2(\delta 2.96,3.12)$ to $\mathrm{C}-1(\delta 197.4)$ and $\mathrm{H}_{2}-4(\delta 2.86$, $3.25)$ to $\mathrm{C}-4 \mathrm{a}(\delta 128.4)$ with the lack of neighbor coupling between the two pairs of non-equivalent methylene protons suggested that the tertiary methyl and hydroxyl group were both attached to the C-3 position. The absolute configuration of the chiral center at C-3 was deduced to be identical with that of the corresponding center of urdamycinone $\mathrm{B},{ }^{4}$ the first $\mathrm{C}-9 \mathrm{C}$-glycoside of tetrangomycin, by comparing their NMR data. The assignment was also supported by the report that all urdamycins were derived on the same biosynthetic pathway. ${ }^{1}$ Differing from urdamycinone $\mathrm{B}$, the placement of the methoxy at C-5 ( $\delta 160.6)$ was confirmed by HMBC correlation from 
Table 1 Physicochemical properties of compounds 1-4

\begin{tabular}{|c|c|c|c|c|}
\hline & $A(1)$ & $B(2)$ & $C(3)$ & $D(4)$ \\
\hline Appearance & Dark yellow powder & Brown powder & Brown powder & Brown powder \\
\hline ESI-MS (m/z) & $707(\mathrm{M}-\mathrm{H})^{-}$ & $481(\mathrm{M}-\mathrm{H})^{-}$ & $693(M-H)^{-}$ & $705(\mathrm{M}-\mathrm{H})^{-}$ \\
\hline \multicolumn{5}{|l|}{$H R-F A B-M S(m / z)$} \\
\hline Found & $709.2871(\mathrm{M}+\mathrm{H})^{+}$ & $483.1656(\mathrm{M}+\mathrm{H})^{+}$ & $695.2628(\mathrm{M}+\mathrm{H})^{+}$ & $707.2759(\mathrm{M}+\mathrm{H})^{+}$ \\
\hline Calculated & $709.2860(\mathrm{M}+\mathrm{H})^{+}$ & $483.1648(\mathrm{M}+\mathrm{H})^{+}$ & $695.2614(\mathrm{M}+\mathrm{H})^{+}$ & $707.2704(\mathrm{M}+\mathrm{H})^{+}$ \\
\hline Retention time (min) & 26.6 & 10.3 & 36.5 & 25.5 \\
\hline
\end{tabular}

the methoxy protons to C-5. The NMR spectra of 1 exhibited the presence of three 6-deoxyhexoses (two of them $O$-glycosidically, one $\mathrm{C}$-glycosidically at the aromatic ring bonded). Especially diagnostic were three methyl doublets $(\delta 1.29,1.25,1.37)$ and three protons in the typical anomeric region $(\delta 5.09,5.00,4.88)$ in the ${ }^{1} \mathrm{H}$ NMR spectrum, and three signals in the region of anomeric carbons $(\delta 99.1,99.5,71.0)$ of the ${ }^{13} \mathrm{C}$ NMR spectrum. The COSY spectrum suggested the sugar substructures from $\mathrm{C}-1^{\prime}$ to $\mathrm{C}-6^{\prime}, \mathrm{C}-1^{\prime \prime}$ to $\mathrm{C}-6^{\prime \prime}$ and $\mathrm{C}-1^{\prime \prime \prime}$ to $\mathrm{C}-3^{\prime \prime \prime}$. In addition, the $\mathrm{HMBC}$ correlations from $\mathrm{CH}_{3}-5^{\prime \prime \prime}$ to $\mathrm{C}-4^{\prime \prime \prime}, \mathrm{C}-5^{\prime \prime \prime}, \mathrm{H}-5^{\prime \prime \prime}$ to $\mathrm{C}-1^{\prime \prime \prime}, \mathrm{C}-4^{\prime \prime \prime}$ and $\mathrm{H}_{2}-3^{\prime \prime \prime}$ to $\mathrm{C}-1^{\prime \prime \prime}, \mathrm{C}-2^{\prime \prime \prime}, \mathrm{C}-4^{\prime \prime \prime}$ ascertained the structure of the terminal sugar. The linkages of the three anomeric carbons were assigned unambiguously by the $\mathrm{HMBC}$ correlations from $\mathrm{H}-1^{\prime}$ to $\mathrm{C}-9, \mathrm{C}-8, \mathrm{C}-10, \mathrm{H}-1^{\prime \prime}$ to $\mathrm{C}-4^{\prime}$ and $\mathrm{H}-1^{\prime \prime \prime}$ to $\mathrm{C}-4^{\prime \prime}$. Furthermore, the configurations of the three sugars (the olivose, the rhodinose and the cinerulose A) were proved to be identical with those in saquayamycin $C^{5}$ by the similarities of their NMR data. The NMR data of 1 were shown in Table 2. Therefore, the overall structure of $\mathbf{1}$ is determined to be 5-methoxy-urdamycinone B moiety joined at the $4^{\prime}$-hydroxyl with the disaccharide, $\alpha$-L-cinerulose A- $(1 \rightarrow 4)-\alpha$-Lrhodinose (Figure 1).

\section{Structure determination of N05WA963B (2)}

N05WA963B (2), was purified as a brown powder with the UV spectrum (Table 1) very similar to that of compound 1 . The high-resolution FAB-MS (m/z 483.1656[M+H $]^{+}$, calcd for 483.1648, $\Delta 0.8 \mathrm{mmu}$ error) showed its molecular formula was $\mathrm{C}_{26} \mathrm{H}_{26} \mathrm{O}_{9}$. In addition, the NMR spectra of $\mathbf{2}$ differed from those of $\mathbf{1}$ only by the absence of signals of cinerulose $\mathrm{A}$ and rhodinose residues (Table 3 ). On the basis of these data, compound 2 is determined to be 5methoxy-urdamycinone B easily (Figure 1).

\section{Structure determination of N05WA963C (3)}

N05WA963C (3), was isolated as a brown powder. Its UV spectrum (Table 1) resembles that of an irradiation product of aquayamycin, dehydrated product IX. ${ }^{2}$ On the basis of the high-resolution FAB-MS $\left(\mathrm{m} / \mathrm{z} 695.2628[\mathrm{M}+\mathrm{H}]^{+}\right.$, calcd for $695.2614, \Delta 1.4 \mathrm{mmu}$ error $)$, the molecular formula was determined to be $\mathrm{C}_{37} \mathrm{H}_{42} \mathrm{O}_{13}$. The signals for two phenolic protons at 13.10 p.p.m. and 13.04 p.p.m. in the ${ }^{1} \mathrm{H}$ NMR spectrum as well as the signals of two hydrogen-bonded quinone carbonyl carbons at $\delta 187.5$ and $\delta 188.0$ in the ${ }^{13} \mathrm{C}$ NMR spectrum indicated the locations of the two hydroxyl groups at the $\alpha$-position of anthraquinone. Furthermore one AB-type system ( $\delta 7.85$ and 7.91 , $J=7.5 \mathrm{~Hz}$ ) and a singlet at $\delta 8.44$ suggested substitutions at the $4 \mathrm{a}-, 5-$, 9-, 10- and 12a-position of the anthraquinone skeleton. The HMBC

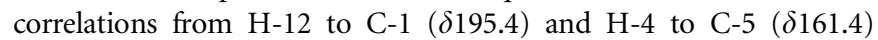

showed the $\mathrm{C}-1(\mathrm{C}=\mathrm{O})$ connecting to the 12a-position and $\mathrm{C}-4\left(\mathrm{CH}_{2}\right)$ connecting to the 4a-position, respectively. The connection between C-2 and C- 1 was established by $\mathrm{HMBC}$ correlations from $\mathrm{H}_{2}-2$ to $\mathrm{C}-1$, C-12a. Cross-peaks from $\mathrm{H}_{2}-2$ to C-3, C-4 and $\mathrm{H}_{3}-13$ to C-2, C-3, C-4 in the HMBC experiment connected the subunits and finally determined the structure of A ring. Thus, the angular frame of aglycone had rearranged to a linear one in compound 3 . The long range correlations from $\mathrm{H}-1^{\prime}$ to $\mathrm{C}-8, \mathrm{C}-9$ and $\mathrm{C}-10$ in the $\mathrm{HMBC}$ spectrum revealed that the pyran ring of the olivose moiety was connected to $\mathrm{C}$ 9 through a C-glycosidic linkage. The patterns of olivose, rhodinose and cinerulose A of $\mathbf{3}$ were identical to those of $\mathbf{1}$ with the comparison of their NMR data. The NMR data of 3 were summarized in Table 4 . The above lines of evidence allowed us to deduce that $\mathbf{3}$ was a novel member of angucycline group unambiguously containing the same aglycone as dehydrated product IX, which was afforded by UV irradiation of aquayamycin in methanol at room temperature. ${ }^{2}$ The structure of $\mathbf{3}$ is in Figure 2.

\section{Structure determination of N05WA963D (4)}

N05WA963D (4) was got as a brown amorphism powder with the UV spectrum (Table 1) that exhibited the presence of a skeleton related to angucycline group. ${ }^{1}$ Its molecular formula, $\mathrm{C}_{38} \mathrm{H}_{42} \mathrm{O}_{13}$, was established from the high resolution FAB-MS (m/z 707. 2759 $[\mathrm{M}+\mathrm{H}]^{+}$, calcd for $707.2704, \Delta 5.5 \mathrm{mmu}$ error). The molecular weight was only $2 \mathrm{mu}$ lower than that of N05WA963A (1). Comparing the NMR data two $s p$ carbons $(\delta 142.8,127.4)$ of 4 were instead of the two $s p$ carbons $(\delta 28.3,33.5)$ of 1 at $2^{\prime \prime \prime}$ and $3^{\prime \prime \prime}$, correspondingly the $\mathrm{C}-1^{\prime \prime \prime}$ and $4^{\prime \prime \prime}$ were shifted upfield to the expectation, apart from that the leaving signals of $\mathbf{1}$ and $\mathbf{4}$ were are very approximate. On the basis of these data, the structure of 4 could be assigned differing from 1 only by the additional dehydrogenations at $2^{\prime \prime \prime}$ and $3^{\prime \prime \prime}$ confidently. Moreover with the similarities of NMR data the patterns of the olivose, the rhodinose and the aculose in $\mathbf{4}$ were conferred to be identical to those in saquayamycin $\mathrm{A}^{5}$ (Table 3 ). Thus the total structure is determined to be 5-methoxy-urdamycinone B moiety joined at the $4^{\prime}$-hydroxyl with the disaccharide, $\alpha$-L-aculose- $(1 \rightarrow 4)-\alpha$ L-rhodinose (Figure 1).

\section{Cytotoxicity to human cancer cell lines}

In the MTT assay, the four compounds $\mathbf{1 - 4}$ and aquayamycin showed growth inhibitory activities on a panel of cancer cell lines such as SW620, K-562, MDA-MB-231, YES-4, T-98 and U251SP (Table 5). The $\mathrm{IC}_{50}$ values of compound 4 against proliferations of above cell lines were $1.0,1.5,2.8,4.0,3.1,3.3 \mu \mathrm{M}$, respectively, which were lower than those of compounds $\mathbf{1}-\mathbf{3}$ and aquayamycin. 
Table 2 NMR data of N05WA963A (1) at $500 \mathrm{MHz}$ in $\mathrm{CDCl}_{3}$

\begin{tabular}{|c|c|c|c|c|}
\hline No. & $\delta_{H}($ mult, $J$ in $H z)$ & $\delta_{C}$ & ${ }^{1} \mathrm{H}_{-}{ }^{1} \mathrm{HCOSY}$ & $H M B C(H$ to $C)$ \\
\hline 1 & & 197.4 & & \\
\hline \multirow[t]{2}{*}{2} & Ha $2.96(1 \mathrm{H}, \mathrm{d}, 14.0)$ & 53.4 & $2-\mathrm{Hb}$ & $1,3,4,12 b, 13$ \\
\hline & $\mathrm{Hb} 3.12(1 \mathrm{H}, \mathrm{d}, 14.0)$ & & $2-\mathrm{Ha}$ & $1,3,4,12 b, 13$ \\
\hline 3 & & 72.4 & & \\
\hline \multirow[t]{2}{*}{4} & Ha $2.86(1 \mathrm{H}, \mathrm{d}, 18.0)$ & 37.6 & $4-\mathrm{Hb}$ & $2,3,4 a, 5,12 b, 13$ \\
\hline & $\mathrm{Hb} 3.25(1 \mathrm{H}, \mathrm{d}, 18.0)$ & & $4-\mathrm{Ha}$ & $2,3,4 a, 5,12 b, 13$ \\
\hline $4 a$ & & 128.4 & & \\
\hline 5 & & 160.6 & & \\
\hline 6 & $7.67(1 \mathrm{H}, \mathrm{s})$ & 108.0 & & $4 a, 5,6 a, 7,12 a$ \\
\hline $6 a$ & & 137.2 & & \\
\hline 7 & & 188.0 & & \\
\hline $7 a$ & & 114.8 & & \\
\hline 8 & & 158.2 & & \\
\hline $8-\mathrm{OH}$ & $12.60(1 \mathrm{H}, \mathrm{s})$ & & & $7,7 a, 8,9$ \\
\hline 9 & & 136.5 & & \\
\hline 10 & $7.87(1 \mathrm{H}, \mathrm{d}, 7.0)$ & 133.8 & 11 & $8,11,11 a, 1^{\prime}$ \\
\hline 11 & $7.68(1 \mathrm{H}, \mathrm{d}, 7.0)$ & 119.5 & 10 & $7 a, 9,10,11 a, 12$ \\
\hline $11 a$ & & 133.8 & & \\
\hline 12 & & 181.7 & & \\
\hline $12 \mathrm{a}$ & & 135.0 & & \\
\hline $12 b$ & & 137.5 & & \\
\hline 13 & $1.51(3 \mathrm{H}, \mathrm{s})$ & 30.5 & & $2,3,4$ \\
\hline 14 & $4.01(3 \mathrm{H}, \mathrm{s})$ & 56.4 & & 5 \\
\hline $1^{\prime}$ & $4.88(1 \mathrm{H}, \mathrm{d}, 11.0)$ & 71.0 & $2^{\prime}-\mathrm{Ha}$ & $8,9,10,2^{\prime}, 3^{\prime}, 5^{\prime}$ \\
\hline \multirow[t]{2}{*}{$2^{\prime}$} & Ha $1.45(1 \mathrm{H}, \mathrm{m})$ & 38.6 & $2^{\prime}-\mathrm{Hb}, 1^{\prime}, 3^{\prime}$ & $9,1^{\prime}, 3^{\prime}, 4^{\prime}$ \\
\hline & $\mathrm{Hb} 2.53(1 \mathrm{H}, \mathrm{m})$ & & $2^{\prime}-\mathrm{Ha}$ & $9,1^{\prime}, 3^{\prime}, 4^{\prime}$ \\
\hline $3^{\prime}$ & $3.82(1 \mathrm{H}, \mathrm{m})$ & 71.5 & $2^{\prime}-\mathrm{Ha}, 2^{\prime}-\mathrm{Hb}, 4^{\prime}$ & $2^{\prime}$ \\
\hline $4^{\prime}$ & $3.08(1 \mathrm{H}, \mathrm{t}, 8.5)$ & 89.0 & $3^{\prime}, 5^{\prime}$ & $3^{\prime}, 5^{\prime}, 6^{\prime}, 1^{\prime \prime}$ \\
\hline $5^{\prime}$ & $3.56(1 \mathrm{H}, \mathrm{m})$ & 74.5 & $4^{\prime}, 6^{\prime}$ & $3^{\prime}, 4^{\prime}, 6^{\prime}$ \\
\hline $6^{\prime}$ & $1.37(3 \mathrm{H}, \mathrm{d}, 6.0)$ & 18.5 & $5^{\prime}$ & $4^{\prime}, 5^{\prime}$ \\
\hline $1^{\prime \prime}$ & $5.00(1 \mathrm{H}, \mathrm{brs})$ & 99.5 & $2^{\prime \prime}-\mathrm{Ha}, 2^{\prime \prime}-\mathrm{Hb}$ & $4^{\prime}, 3^{\prime \prime}, 5^{\prime \prime}$ \\
\hline \multirow[t]{2}{*}{$2^{\prime \prime}$} & Ha $1.68(1 \mathrm{H}, \mathrm{m})$ & 25.2 & $1^{\prime \prime}, 2^{\prime \prime}-\mathrm{Hb}$ & $1^{\prime \prime}, 4^{\prime \prime}$ \\
\hline & $\mathrm{Hb} 2.11(1 \mathrm{H}, \mathrm{m})$ & & $1^{\prime \prime}, 2^{\prime \prime}-\mathrm{Ha}$ & $1^{\prime \prime}, 4^{\prime \prime}$ \\
\hline \multirow[t]{2}{*}{$3^{\prime \prime}$} & Ha $1.91(1 \mathrm{H}, \mathrm{m})$ & 24.5 & $3^{\prime \prime}-\mathrm{Hb}, 4^{\prime \prime}$ & $1^{\prime \prime}, 2^{\prime \prime}, 4^{\prime \prime}, 5^{\prime \prime}$ \\
\hline & $\mathrm{Hb} 2.11(1 \mathrm{H}, \mathrm{m})$ & & $3 "-\mathrm{Ha}$ & $1^{\prime \prime}, 2^{\prime \prime}, 4^{\prime \prime}, 5^{\prime \prime}$ \\
\hline $4^{\prime \prime}$ & $3.69(1 \mathrm{H}, \mathrm{brs})$ & 74.7 & $3^{\prime \prime}-\mathrm{Ha}, 5^{\prime \prime}$ & $2^{\prime \prime}, 1^{\prime \prime \prime}$ \\
\hline $5^{\prime \prime}$ & $4.23(1 \mathrm{H}, \mathrm{m})$ & 68.0 & $4^{\prime \prime}, 6^{\prime \prime}$ & $1^{\prime \prime}, 4^{\prime \prime}, 6^{\prime \prime}$ \\
\hline $6^{\prime \prime}$ & $1.25(3 \mathrm{H}, \mathrm{d}, 6.5)$ & 16.9 & $5^{\prime \prime}$ & $4^{\prime \prime}, 5^{\prime \prime}$ \\
\hline $1^{\prime \prime \prime}$ & $5.09(1 \mathrm{H}, \mathrm{t}, 5.5)$ & 99.1 & $2^{\prime \prime \prime}-\mathrm{Ha}, 2^{\prime \prime \prime}-\mathrm{Hb}$ & $4^{\prime \prime}, 3^{\prime \prime \prime}, 5^{\prime \prime \prime}$ \\
\hline \multirow[t]{2}{*}{$2^{\prime \prime \prime}$} & $\mathrm{Ha} 2.14(1 \mathrm{H}, \mathrm{m})$ & 28.3 & $2^{\prime \prime \prime}-\mathrm{Hb}, 1^{\prime \prime \prime}$ & $1^{\prime \prime \prime}, 3^{\prime \prime \prime}, 4^{\prime \prime \prime}$ \\
\hline & $\mathrm{Hb} 2.37(1 \mathrm{H}, \mathrm{m})$ & & $2^{\prime \prime \prime}-\mathrm{Ha}, 1^{\prime \prime \prime}$ & $1^{\prime \prime \prime}, 3^{\prime \prime \prime}, 4^{\prime \prime \prime}$ \\
\hline $3^{\prime \prime \prime}$ & $2.50(2 \mathrm{H}, \mathrm{m})$ & 33.5 & $2^{\prime \prime \prime}-\mathrm{Ha}, 2^{\prime \prime \prime}-\mathrm{Hb}$ & $1^{\prime \prime \prime}, 2^{\prime \prime \prime}, 4^{\prime \prime \prime}$ \\
\hline $4^{\prime \prime \prime}$ & & 210.8 & & \\
\hline $5^{\prime \prime \prime}$ & $4.33(1 \mathrm{H}, \mathrm{q}, 7.0)$ & 71.1 & $6^{\prime \prime \prime}$ & $1^{\prime \prime \prime}, 4^{\prime \prime \prime}, 6^{\prime \prime \prime}$ \\
\hline $6^{\prime \prime \prime}$ & $1.29(3 \mathrm{H}, \mathrm{d}, 7.0)$ & 14.8 & $5^{\prime \prime \prime}$ & $4^{\prime \prime \prime}, 5^{\prime \prime \prime}$ \\
\hline
\end{tabular}

$\delta$ in p.p.m. relative to internal TMS.

\section{DISCUSSION}

The angucycline family of antibiotics is a large and ever-growing group of secondary metabolites of microbial origin containing more than 100 members. Structurally, these compounds consist of polyketide origin possessing benz $[\alpha]$ anthracene nuclei and several C-glycosidic or $O$-glycosidic linkages. ${ }^{1}$ In addition to diverse structural features, they also display a broad range of biological activities, such as antitumor, ${ }^{7,8}$ enzyme inhibitory, ${ }^{9,10}$ blood platelet aggregation inhibitory ${ }^{11}$ and antimicrobial..$^{8,12}$ On the other hand, anthracyclines are widely used as antitumoral agents, for example, doxorubicin with $\mathrm{ID}_{50}$ of $1.02 \mu \mathrm{M}$ against K-562 cells in vitro. ${ }^{13}$ Our findings provide

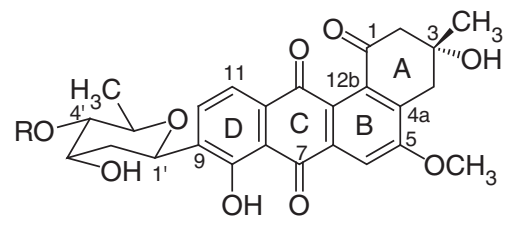

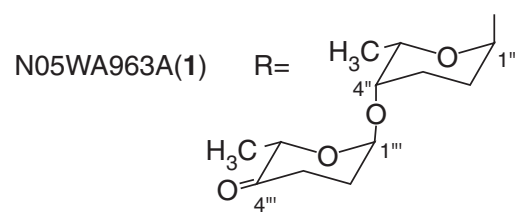

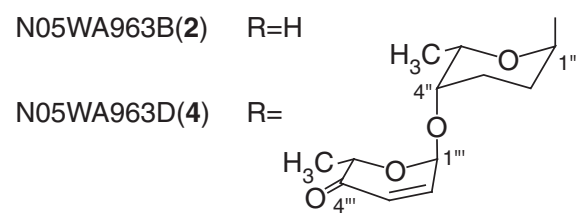

Figure 1 Structures of N05WA963A (1), N05WA963B (2) and N05WA963D (4)

four novel chemical entities: three angucyclines and one anthracycline with cytotoxicity against six cancer cell lines: SW620, K-562, MDAMB-231, YES-4, T-98 and U251SP. The activity of compound 4 in our assay was higher than those of compounds $\mathbf{1 - 3}$ and aquayamycin. The preliminary bioactivity evaluation indicated that these compounds might serve as useful leads in the development of new anti-cancer pharmaceutical agents.

\section{METHODS}

\section{Reagents and cell lines}

MTT was purchased from Sigma (St Louis, MO, USA). Human colorectal cancer cell line SW620, human leukemia cell line K-562, human breast cancer cell line MDA-MB-231 were purchased from American Type Culture Collection (Rockvill, MD, USA). Esophageal carcinoma cell line YES-4, human brain nerve colloid tumor cell line T-98 and human brain nerve colloid tumor cell line U251SP were obtained from medical college of Japan Chiba University. RPMI1640 medium was purchased from Sigma. Aquayamycin was supplied by the antibiotics library in New Drug Research and Development Center, North China Pharmaceutical Group Corporation.

\section{Analytical measurement}

Observation of mycelia was performed using a CX31RTSF microscope (Olympus Corporation, Tokyo, Japan). UV spectra were recorded with a Lambda 35 UV/VIS spectrometer (Perkin Elmer Life Sciences, Turku, Finland). HR-FABMS were measured with a Thermo scientific LTQ FT mass spectrometer (Thermo fisher scientific, Somerset, New Jersey, USA). The 1D and 2D NMR spectra were measured on a Varian Inova-500 spectrometer (Varian, Palo Alto, CA, USA) using tetramethylsilicane (TMS) as an internal standard. HPLC was performed using a Waters HPLC system equipped with a 996 photodiode array detector (Waters, Milford, MA, USA). Absorbance values in MTT test were recorded with a Wallac Victor ${ }^{2} 1420$ plate reader (Pekin Elmer Life Sciences, Turku, Finland).

\section{Taxonomic studies}

The investigative strain N05WA963 was isolated from a soil sample collected in Yunnan Province, China. For morphological observation, strain N05WA963 was cultured on ISP2 media at $28^{\circ} \mathrm{C}$ for 14 to 21 days. Mycelia were observed using a light microscope.

For phylogenetic study, fresh cells were ground to get a uniform suspension. Preparation of genomic DNA and amplification of the 16S ribosomal DNA 
Table 3 NMR data of N05WA963B (2), D (4) at $500 \mathrm{MHz}$ in $\mathrm{CDCl}_{3}$

\begin{tabular}{|c|c|c|c|c|}
\hline \multirow[b]{2}{*}{ No. } & \multicolumn{2}{|l|}{ N05WA963B (2) } & \multicolumn{2}{|l|}{ N05WA963D (4) } \\
\hline & $\delta_{H}($ mult, $\mathrm{J}$ in $\mathrm{Hz}$ ) & $\delta_{C}$ & $\delta_{H}($ mult, $J$ in $\mathrm{Hz})$ & $\delta_{C}$ \\
\hline 1 & & 197.4 & & 197.6 \\
\hline \multirow[t]{2}{*}{2} & Ha $2.98(1 \mathrm{H}, \mathrm{d}, 14.0)$ & 53.5 & Ha $2.96(1 \mathrm{H}, \mathrm{d}, 14.0)$ & 53.3 \\
\hline & $\mathrm{Hb} 3.13(1 \mathrm{H}, \mathrm{d}, 14.0)$ & & $\mathrm{Hb} 3.13(1 \mathrm{H}, \mathrm{d}, 14.0)$ & \\
\hline 3 & & 72.5 & & 72.2 \\
\hline \multirow[t]{2}{*}{4} & Ha $2.90(1 \mathrm{H}, \mathrm{d}, 18.0)$ & 37.6 & Ha $2.86(1 \mathrm{H}, \mathrm{d}, 18.0)$ & 37.5 \\
\hline & $\mathrm{Hb} 3.28(1 \mathrm{H}, \mathrm{d}, 18.0)$ & & $\mathrm{Hb} 3.26(1 \mathrm{H}, \mathrm{d}, 18.0)$ & \\
\hline $4 a$ & & 128.5 & & 128.4 \\
\hline 5 & & 160.7 & & 160.7 \\
\hline 6 & $7.73(1 \mathrm{H}, \mathrm{s})$ & 108.1 & $7.69(1 \mathrm{H}, \mathrm{s})$ & 108.0 \\
\hline $6 a$ & & 137.2 & & 137.4 \\
\hline 7 & & 188.0 & & 188.0 \\
\hline $7 a$ & & 114.8 & & 114.8 \\
\hline 8 & & 158.2 & & 158.2 \\
\hline $8-\mathrm{OH}$ & $12.63(1 \mathrm{H}, \mathrm{s})$ & & $12.61(1 \mathrm{H}, \mathrm{s})$ & \\
\hline 9 & & 136.3 & & 136.4 \\
\hline 10 & $7.88(1 \mathrm{H}, \mathrm{d}, 8.0)$ & 133.9 & $7.87(1 \mathrm{H}, \mathrm{d}, 7.0)$ & 133.8 \\
\hline 11 & $7.69(1 \mathrm{H}, \mathrm{d}, 8.0)$ & 119.6 & $7.67(1 \mathrm{H}, \mathrm{d}, 7.0)$ & 119.5 \\
\hline $11 a$ & & 133.9 & & 133.8 \\
\hline 12 & & 181.8 & & 181.9 \\
\hline $12 a$ & & 135.1 & & 135.0 \\
\hline $12 b$ & & 137.6 & & 137.5 \\
\hline 13 & $1.53(3 \mathrm{H}, \mathrm{s})$ & 30.6 & $1.52(3 \mathrm{H}, \mathrm{s})$ & 30.5 \\
\hline 14 & $4.04(3 \mathrm{H}, \mathrm{s})$ & 56.4 & $4.01(3 \mathrm{H}, \mathrm{s})$ & 56.4 \\
\hline $1^{\prime}$ & $4.92(1 \mathrm{H}, \mathrm{d}, 11.0)$ & 71.2 & $4.88(1 \mathrm{H}, \mathrm{d}, 11.0)$ & 71.0 \\
\hline \multirow[t]{2}{*}{$2^{\prime}$} & Ha $1.48(1 \mathrm{H}, \mathrm{m})$ & 39.4 & Ha $1.45(1 \mathrm{H}, \mathrm{m})$ & 38.6 \\
\hline & $\mathrm{Hb} 2.51(1 \mathrm{H}, \mathrm{m})$ & & $\mathrm{Hb} 2.53(1 \mathrm{H}, \mathrm{m})$ & \\
\hline $3^{\prime}$ & $3.83(1 \mathrm{H}, \mathrm{m})$ & 73.2 & $3.82(1 \mathrm{H}, \mathrm{m})$ & 71.5 \\
\hline $4^{\prime}$ & $3.20(1 \mathrm{H}, \mathrm{t}, 9.0)$ & 78.1 & $3.07(1 \mathrm{H}, \mathrm{t}, 8.5)$ & 88.9 \\
\hline $5^{\prime}$ & $3.50(1 \mathrm{H}, \mathrm{m})$ & 75.9 & $3.56(1 \mathrm{H}, \mathrm{m})$ & 74.5 \\
\hline $6^{\prime}$ & $1.42(3 \mathrm{H}, \mathrm{d}, 6.0)$ & 18.1 & $1.37(3 \mathrm{H}, \mathrm{d}, 6.0)$ & 18.4 \\
\hline $1^{\prime \prime}$ & & & $5.00(1 \mathrm{H}, \mathrm{brs})$ & 99.5 \\
\hline \multirow[t]{2}{*}{$2^{\prime \prime}$} & & & Ha $1.69(1 \mathrm{H}, \mathrm{m})$ & 25.1 \\
\hline & & & Hb $2.09(1 \mathrm{H}, \mathrm{m})$ & \\
\hline \multirow[t]{2}{*}{$3^{\prime \prime}$} & & & Ha $1.92(1 \mathrm{H}, \mathrm{m})$ & 24.3 \\
\hline & & & $\mathrm{Hb} 2.11(1 \mathrm{H}, \mathrm{m})$ & \\
\hline $4^{\prime \prime}$ & & & $3.72(1 \mathrm{H}, \mathrm{m})$ & 76.2 \\
\hline $5^{\prime \prime}$ & & & $4.24(1 \mathrm{H}, \mathrm{m})$ & 67.7 \\
\hline $6^{\prime \prime}$ & & & $1.28(3 \mathrm{H}, \mathrm{d}, 6.0)$ & 16.9 \\
\hline $1^{\prime \prime \prime}$ & & & $5.27(1 \mathrm{H}, \mathrm{d}, 3.5)$ & 95.2 \\
\hline $2^{\prime \prime \prime}$ & & & $6.88(1 \mathrm{H}, \mathrm{dd}, 10.0,3.5)$ & 142.8 \\
\hline $3^{\prime \prime \prime}$ & & & $6.11(1 \mathrm{H}, \mathrm{d}, 10.0)$ & 127.4 \\
\hline $4^{\prime \prime \prime}$ & & & & 196.7 \\
\hline $5^{\prime \prime \prime}$ & & & $4.58(1 \mathrm{H}, \mathrm{q}, 6.5)$ & 70.6 \\
\hline $6^{\prime \prime \prime}$ & & & $1.38(3 \mathrm{H}, \mathrm{d}, 6.5)$ & 15.1 \\
\hline
\end{tabular}

$\delta$ in p.p.m. relative to internal TMS.

were performed according to the procedure described by $\mathrm{Xu}^{6}{ }^{6}$ The primer pair 27f (5'-AGAGTTTGATCCTGGCTCAG- $\left.3^{\prime}\right)$ and 1492r (5'-TACGGCTACCTT GTTACGACTT- $3^{\prime}$ ) was used for amplification of the almost complete $16 \mathrm{~S}$ ribosomal RNA gene.

\section{Fermentation of strain N05WA963}

Spores obtained from a well-sporulated slant of this organism were inoculated to the medium which consisted of soluble starch $2.4 \%$, glucose $0.1 \%$, peptone $0.3 \%$, beef paste $0.3 \%$, yeast extracts $0.5 \%$ and $\mathrm{CaCO}_{3} 0.4 \%$ (pH7.0 before sterilization), and were shake-cultured at $28^{\circ} \mathrm{C}$ for $72 \mathrm{~h}$ in 500 -ml Erlenmeyer flasks containing $100 \mathrm{ml}$ of the medium described above. Thereafter, $5 \mathrm{ml}$

Table 4 NMR data of N05WA963C (3) at $500 \mathrm{MHz}$ in $\mathrm{CDCl}_{3}$

\begin{tabular}{llrl}
\hline No. & $\delta_{H}($ mult, J in Hz) & \multicolumn{1}{c}{$\delta_{C}$} & HMBC (H to C) \\
\hline 1 & & 195.4 & \\
2 & Ha 2.81 (1H, d, 16.5) & 51.6 & $1,3,4,12 \mathrm{a}, 13$ \\
& Hb 2.92 (1H, d, 16.5) & & $1,3,4,12 \mathrm{a}, 13$ \\
3 & & 71.4 & \\
4 & Ha 3.05 (1H, d, 18.5) & 37.0 & $2,3,5,12 \mathrm{a}$ \\
& Hb 3.37 (1H, d, 18.5) & & $2,3,5,12 \mathrm{a}$
\end{tabular}

$4 a$

5

$5-\mathrm{OH}$

$5 a$

6

$6 a$

7

8

9

10

10-OH

$10 a$

11

$11 a$

12

$12 \mathrm{a}$

13

$1^{\prime}$

$2^{\prime}$

$3^{\prime}$

$4^{\prime}$

$5^{\prime}$

$6^{\prime}$

$1^{\prime \prime}$

2"

$3^{\prime \prime}$

$4 "$

$5^{\prime \prime}$

6"

$1^{\prime \prime \prime}$

$2^{\prime \prime \prime}$

$3^{\prime \prime \prime}$

$4^{\prime \prime \prime}$

$5^{\prime \prime \prime}$

$6^{\prime \prime \prime}$
137.4

161.4

$13.10(1 \mathrm{H}, \mathrm{s})$

$7.85(1 \mathrm{H}, \mathrm{d}, 7.5)$

$7.91(1 \mathrm{H}, \mathrm{d}, 7.5)$

$13.04(1 \mathrm{H}, \mathrm{s})$

$\begin{array}{lrc} & 115.5 & \\ & 187.5 & \\ & 131.0 & \\ 8.44(1 \mathrm{H}, \mathrm{s}) & 116.8 & 1,4 \mathrm{a}, 5 \mathrm{a}, 11 \\ & 137.1 & \\ 1.55(3 \mathrm{H}, \mathrm{s}) & 30.1 & 2,3,4\end{array}$

$4.90(1 \mathrm{H}, \mathrm{d}, 11.0) \quad 71.1$

Ha $1.45(1 \mathrm{H}, \mathrm{m}) \quad 38.5$

$\mathrm{Hb} 2.53(1 \mathrm{H}, \mathrm{m})$

$3.84(1 \mathrm{H}, \mathrm{m})$

$3.07(1 \mathrm{H}, \mathrm{m})$

$3.56(1 \mathrm{H}, \mathrm{m})$

$1.38(3 \mathrm{H}, \mathrm{d}, 5.5)$

$5.01(1 \mathrm{H}, \mathrm{m})$

Ha $1.68(1 \mathrm{H}, \mathrm{m})$

$\mathrm{Hb} 2.09(1 \mathrm{H}, \mathrm{m})$

Ha $1.92(1 \mathrm{H}, \mathrm{m})$ $8,9,10,2^{\prime}, 3^{\prime}, 5^{\prime}$

$9,1^{\prime}, 3^{\prime}, 4^{\prime}$

9, $1^{\prime}, 3^{\prime}, 4^{\prime}$

$4^{\prime}$

$3^{\prime}, 5^{\prime}, 6^{\prime}, 1^{\prime \prime}$

$4^{\prime}, 5^{\prime}$

$4^{\prime}, 3^{\prime \prime}, 5^{\prime \prime}$

$1^{\prime \prime}, 4^{\prime \prime}$

$1^{\prime \prime}, 4^{\prime \prime}$

24.5

74.7

68.0

17.0

99.1

28.3

33.5

210.9

71.1

14.8
$\mathrm{Hb} 2.10(1 \mathrm{H}, \mathrm{m})$

$3.69(1 \mathrm{H}, \mathrm{brs})$

$4.22(1 \mathrm{H}, \mathrm{m})$

$1.26(3 \mathrm{H}, \mathrm{d}, 6.5)$

$5.09(1 \mathrm{H}, \mathrm{m})$

Ha $2.10(1 \mathrm{H}, \mathrm{m})$

$\mathrm{Hb} 2.38(1 \mathrm{H}, \mathrm{m})$

$2.50(2 \mathrm{H}, \mathrm{m})$

$4.33(1 \mathrm{H}, \mathrm{q}, 6.5)$

$1.29(3 \mathrm{H}, \mathrm{d}, 6.5)$

$1^{\prime \prime}$
$2^{\prime \prime}, 1^{\prime \prime \prime}$
$4^{\prime \prime}, 6^{\prime \prime}$
$4^{\prime \prime}, 5^{\prime \prime}$
$4^{\prime \prime}, 5^{\prime \prime \prime}$
$1^{\prime \prime \prime}, 3^{\prime \prime \prime}, 4^{\prime \prime \prime}$
$1^{\prime \prime \prime}, 3^{\prime \prime \prime}, 4^{\prime \prime \prime}$
$1^{\prime \prime \prime}, 2^{\prime \prime \prime}, 4^{\prime \prime \prime}$
$1^{\prime \prime \prime}, 4^{\prime \prime \prime}, 6^{\prime \prime \prime}$
$4^{\prime \prime \prime}, 5^{\prime \prime \prime}$

$\delta$ in p.p.m. relative to internal TMS. portions of this seed culture were transferred into 500-ml Erlenmeyer flasks, which contained $100 \mathrm{ml}$ of a ferment medium consisting of soluble starch $4.0 \%$, bean powder $2.0 \%$, corn steep liquor $0.5 \%, \mathrm{KH}_{2} \mathrm{PO}_{4} 0.05 \%, \mathrm{FeSO}_{4} \cdot 7 \mathrm{H}_{2} \mathrm{O}$ $0.05 \%, \mathrm{KCl} 0.03 \%$ ( $\mathrm{pH} 6.5$ before sterilization) and then were incubated at $28{ }^{\circ} \mathrm{C}$ for $144 \mathrm{~h}$ on a rotary shaker at 220 r.p.m.

\section{Isolation procedure for angucycline group compounds}

The fermentation broth of flasks was harvested to total volume of 4.01 and centrifuged at 3000 r.p.m. for $15 \mathrm{~min}$ to separate filtrate and mycelia. The filtrate was extracted with ethyl acetate. The mycelia were extracted with $70 \%$ acetone. After the insoluble mycelia were removed by centrifugation, the 


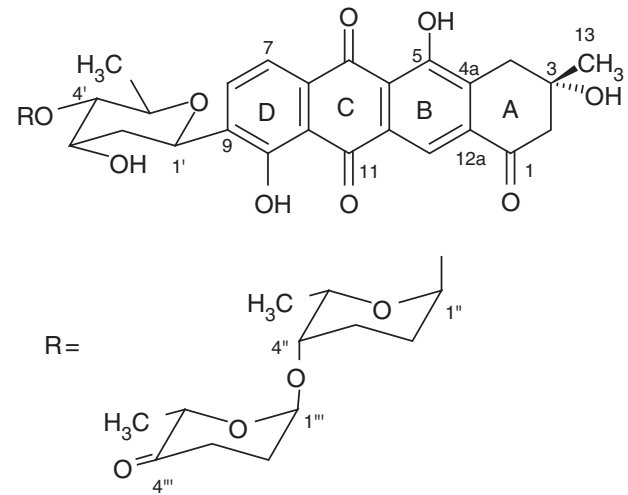

Figure 2 Structure of N05WA963C (3).

Table 5 Cytotoxicity of compounds $1-4$ and aquayamycin on cancer cell lines

\begin{tabular}{lcrrrrr}
\hline & \multicolumn{7}{c}{$I_{50}(\mu \mathrm{m})$} \\
\cline { 2 - 7 } Compound & SW620 & K-562 & MDA-MB-231 & YES-4 & T-98 & U251SP \\
\hline N05WA963A (1) & 4.4 & 19.7 & 9.8 & 7.9 & 5.3 & 5.6 \\
N05WA963B (2) & 7.1 & 20.7 & 16.6 & 8.5 & 21.4 & 10.3 \\
N05WA963C (3) & 1.5 & 11.4 & 6.5 & 4.5 & 5.9 & 4.1 \\
N05WA963D (4) & 1.0 & 1.5 & 2.8 & 4.0 & 3.1 & 3.3 \\
Aquayamycin & 6.0 & 21.4 & 20.5 & 4.3 & 11.8 & 23.4 \\
\hline
\end{tabular}

acetone was evaporated and the remaining aqueous solution was extracted with ethyl acetate. The all above ethyl acetate layers were combined and concentrated to yield a brown residue $(1.8 \mathrm{~g})$. The residue was subjected to a silica gel flash column and eluted with a gradient of chloroform-methanol from 100:1 to 10:1. The fractions, including active materials, were collected and evaporated to afford $0.5 \mathrm{~g}$ brown residue. The residue was applied on a preparative reversedphase-HPLC column (detection: UV at $254 \mathrm{~nm}$; column: Phenomenex (Torrance, CA, USA) $\mathrm{C}_{18}, 10 \mu \mathrm{m}, 21.2 \times 250 \mathrm{~mm}$; mobile phase: a linear gradient of $30 \%$ acetonitrile-water to $60 \%$ acetonitrile-water over $10 \mathrm{~min}$, then $60 \%$ acetonitrile-water over $30 \mathrm{~min}$; flow rate: $16.0 \mathrm{ml} \mathrm{min}^{-1}$ ) to obtain four pure compounds: $9.1 \mathrm{mg}$ of N05WA963A (1), $17.0 \mathrm{mg}$ of N05WA963B (2), $15.1 \mathrm{mg}$ of N05WA963C (3) and $13.4 \mathrm{mg}$ of N05WA963D (4), respectively. The retention time of each compound is shown in Table 1.

\section{Assay for cytotoxicity on cancer cell lines}

Cytotoxicity of the compounds was examined using a MTT method. ${ }^{14}$ All cell lines were routinely cultured and passaged in RPMI1640 medium supplemented with $10 \%$ new calf serum and $2 \mathrm{~mm} \mathrm{L-glutamine}$ at $37^{\circ} \mathrm{C}$ in a humidified atmosphere of $5 \% \mathrm{CO}_{2}$. For cytotoxicity assay, $100 \mu \mathrm{l}$ of SW620, K-562, MDAMB-231, YES-4, T-98 or U251SP cells were seeded in 96-well plates at $1 \times 10^{4}$ cells per well and incubated with series of dilution of test compounds for 3 days. After treatment, cells were then incubated with $5 \mathrm{mg} \mathrm{ml}^{-1}$ MTT solution for $4 \mathrm{~h}$. In all, $100 \mu \mathrm{l}$ of $10 \%$ sodium dodecyl sulfate solution was added to the culture and the absorbance at $570 \mathrm{~nm}$ was measured on the plate reader. The $\mathrm{IC}_{50}$ values were obtained from the inhibition curve against various concentrations of test compounds.

\section{ACKNOWLEDGEMENTS}

This work was supported by the grants from the National Basic Research Project of China (Grant 2009CB526513), National New Drug Research and Development Project (Grant 2008ZX09401-05, 2010ZX09401-403). We are grateful to the colleagues from the New Drug Research and Development Center, North China Pharmaceutical Group for their support especially Mr Dong Guo and Ms Shiqing Zhu for NMR and MS measurements and $\mathrm{Mr}$ Yunlong $\mathrm{Mu}$, Ms Chaojing Cai and Ms Yueqi Shan for the actinomycete collection and fermentation. We thank Dr Mei Dong (School of Pharmaceutical Sciences, Hebei Medical University) for cytotoxicity assay in part.

1 Rohr, J. \& Thicricke, R. Augucycline group antibiotic. Nat. Prod. Rep. 9, 103-137 (1992).

2 Sezaki, M., Kondo, S., Maeda, K. \& Umezawa, H. The structure of aquayamycin. Tetrahedron 26, 5171-5190 (1970).

3 Drautz, H. \& Zahner, H. Metabolic products of microorganisms. 234 Urdamycins, new angucycline antibiotics from Streptomyces fradiae. I. Isolation, characterization and biological properties. J. Antibiot. 39, 1657-1669 (1986).

4 Rohr, J. \& Zeeck, A. Metabolic products of microorganisms. 240. urdamycins, new angucycline antibiotics from Streptomyces fradiae. II. Structural studies of urdamycins B to F. J. Antibiot. 40, 459-467 (1987).

5 Uchida, T. et al. Saquayamycins, new aquayamycin-group antibiotics. J. Antibiot. $\mathbf{3 8}$ 1171-1181 (1985)

6 Xu, L. H., Li, W. J., Liu, Z. H. \& Jiang, C. L. Actinomycete Systematic: Principle, Methods and Practice 93-149 (Science Press, Beijing, 2007).

7 Abdelfattah, M. S., Kharel, M. K., Hitron, J. A., Baig, I. \& Rohr, J. Moromycins A and B, isolation and structure elucidation of $\mathrm{C}$-glycosylangucycline-type antibiotics from Streptomyces sp. KYO02. J. Nat. Prod. 71, 1569-1573 (2008).

8 Maruna, M. et al. Isolation, structure elucidation and biological activity of angucycline antibiotics from an epiphytic yew Streptomycete. J. Basic Microbiol. 50, 135-142 (2010).

9 Alvi, K. A., Baker, D. D., Stienecker, V., Hosken, M. \& Nair, B. G. Identification of inhibitors of inducible nitric oxide synthase from microbial extracts. J. Antibiot. 53, 496-501 (2000).

10 Sekizawa, R. et al. Isolation of novel saquayamycins as inhibitors of farnesyl-protein trasferase. J. Antibiot. 49, 487-490 (1996).

11 Kawashima, A. et al. PI-083, a new platelet aggregation inhibitor. J. Antibiot. 41, 1913-1914 (1988)

12 Abdelfattah, M., Maskey, R. P., Asolkar, R. N., Grun, W. I. \& Laatsch, H. Seitomycin: isolation, structure elucidation and biological activity of a new angucycline antibiotic from a terrestrial Streptomycete. J. Antibiot. 56, 539-542 (2003).

13 Biscardi, M. et al. In vitro antileukemic effect of a new anthracycline analogue, MEN 11079. Leuk. Res. 27, 1125-1134 (2003).

14 Mosmann, T. Rapid colorimetric assay for cellular growth and survival: application to proliferation and cytotoxicity assays. J. Immunol. Meth. 65, 55-63 (1983). 